\title{
Measurement of dietary exposure: a challenging problem which may be overcome thanks to metabolomics?
}

\author{
Gaëlle Favé · M. E. Beckmann · J. H. Draper · \\ J. C. Mathers
}

Received: 16 March 2009/Accepted: 18 March 2009/Published online: 2 April 2009

(c) Springer-Verlag 2009

\begin{abstract}
The diet is an important environmental exposure, and its measurement is an essential component of much health-related research. However, conventional tools for measuring dietary exposure have significant limitations being subject to an unknown degree of misreporting and dependent upon food composition tables to allow estimation of intakes of energy, nutrients and non-nutrient food constituents. In addition, such tools may be inappropriate for use with certain groups of people. As an alternative approach, the recent techniques of metabolite profiling or fingerprinting, which allows simultaneous monitoring of multiple and dynamic components of biological fluids, may provide metabolic signals indicative of food intake. Samples can be analysed through numerous analytical platforms, followed by multivariate data analysis. In humans, metabolomics has been applied successfully in pharmacology, toxicology and medical screening, but nutritional metabolomics is still in its infancy. Biomarkers of a small number of specific foods and nutrients have been developed successfully but less targeted and more high-throughput methods, that do not need prior knowledge of which signals might be discriminatory, and which may allow a more global characterisation of dietary intake, remain to be tested. A proof a principle project (the MEDE Study) is currently underway in our laboratories to test
\end{abstract}

\footnotetext{
G. Favé $(\bowtie)$ · J. C. Mathers

Human Nutrition Research Centre, Institute for Ageing and Health,

Newcastle University, Framlington Place,

Newcastle upon Tyne NE2 4HH, UK

e-mail: Gaelle.Fave@newcastle.ac.uk

M. E. Beckmann · J. H. Draper

Institute of Biological, Environmental and Rural Sciences,

Aberystwyth University, Aberystwyth,

Ceredigion SY23 3DA, UK
}

the hypothesis that high-throughput, non-targeted metabolite fingerprinting using flow injection electrospray mass spectrometry can be applied to human biofluids (blood and urine) to characterise dietary exposure in humans.

Keywords Dietary exposure - Human studies . Mass spectrometry · Metabolite fingerprinting . Nutritional metabolomics .

Multivariate supervised classifications

\section{Introduction}

The diet is an important environmental exposure, and many dietary factors (nutrients and non-nutrients) are associated with disease prevention or causation [5]. The measurement of habitual dietary intake is thus an essential component of much health-related research, which must be both accurate and applicable to very large numbers of free-living individuals. This makes measurement of dietary exposure one of the most challenging problems in nutrition.

\section{Problems with measuring dietary exposure}

Conventional tools for collecting quantitative information on dietary exposure, such as diet diaries, food frequency questionnaires and $24 \mathrm{~h}$ recalls can be unreliable methods for characterising and quantifying eating behaviour. These tools are associated with both random and systematic errors arising from the assessment of the nature and frequency of food consumption and of portion size, daily variation in intake, the failure to report usual diet (due to changes in habits whilst taking part in an investigation or misreporting of food choice or amount) and the use of food tables to 
convert intakes of foods to intakes of energy, nutrients and other food components [5]. Bingham and collaborators investigated the accuracy of several dietary assessment methods in the UK arm of the European Prospective Investigation of Cancer and Nutrition (EPIC) by comparing 16 days of weighed records, a food frequency questionnaire (FFQ), a 24-h recall method and a 7-day food diary [6]. Their data showed that, when compared with weighed records, the FFQ tended to over-estimate almost all intakes whereas the 24-h recall under-estimated carbohydrates, vitamin $\mathrm{C}$ and alcohol intakes and the food diary overestimated fat intake but under-estimated intakes of carbohydrates and calcium [6]. In addition, such conventional tools are inappropriate and/or unreliable for certain groups such as the obese or elderly people, whose self-reported energy intakes tend to be underestimated, as assessed by energy expenditure measurements using the doubly labelled water method [50,63].

Inaccurate measurement of dietary exposure may make it difficult or even impossible to detect correlations between dietary exposure and disease risk. The follow-up study of markers of aflatoxin exposure in relation to liver cancer undertaken by Qian and collaborators is a good example. The relative risk (RR) of cancer, from aflatoxin consumption for individuals with high dietary exposure, was only 0.9 and was not significant when exposure was assessed by frequency of consumption of 45 foods, but the RR was 59.4 (and highly significant) when exposure was measured using biomarkers in urine samples [46]. Limitations in the accuracy and/or precision of measurements of dietary intake may help explain the conflicting results about the protective effect of micronutrients such as antioxidant vitamins in respect of risk of cancers or of cardiovascular disease. For example, the associations between breast cancer risk and dietary carotenoids, retinol, vitamin $\mathrm{C}$ and tocopherols remain uncertain, as demonstrated by inconsistent results from studies using, even validated, FFQ [59].

In an attempt to overcome the problems with measuring dietary exposure with self-reported methods, a number of biomarkers have been developed which can be used to validate intake estimates or to act as surrogates for intake measurements. Such biomarkers include the use of urinary output of potassium for potassium intakes. The correlation between intake and excretion can be very good (at least $0.7)$, even when dietary intakes are calculated from food tables rather than analysed, as long as sufficient $24 \mathrm{~h}$ urine samples are obtained [5]. Similarly, there are good correlations between individual estimates of protein intake and the 24-h urine nitrogen output provided, that, the completeness of the urine collection is checked using an independent marker e.g. $p$-aminobenzoic acid [5]. Further, the fatty acid composition of blood lipids or of adipose tissue can act as a biomarker of fatty acid intakes. Good correlations exist between concentrations of pentadecanoic acid (C15:0) and intake estimates of fats from milk or dairy products $[51,66]$. When total fatty acid intakes were estimated [44], the correlation with the fatty acid composition of subcutaneous tissue remained good $(R=0.50)$ for polyunsaturated fatty acids, but not for mono-unsaturated or saturated fatty acids $(R=0.22$ and 0.24 , respectively) probably because the latter can be synthesised in the tissues as well as being obtained from the diet. Whilst such individual biomarkers are valuable, they are not suitable for describing dietary exposure as a whole because they reflect only a very small range of food constituents. Thus, there is an urgent need to develop an alternative, non-subjective tool that can be used to assess the totality of dietary exposure and which could be applied to relatively large numbers of individuals at relatively low cost.

\section{Advent of metabolomics approaches}

Foods contain thousands of compounds which, upon digestion and metabolism, give rise to the metabolites present in body fluids such as blood and urine. In theory, it should be possible to distinguish which foods have been eaten and in what amounts from an assessment of the metabolites in these fluids. However, digestion, transport, storage, metabolism and excretion of food constituents is a complex and dynamic process resulting in a myriad of different metabolites present in a very wide range of concentrations. Until very recently, this complexity has meant that it was virtually impossible to design a strategy for assessing dietary exposure which would have the technological reach to address the heterogeneity of metabolites and have sufficient capacity to cope with large numbers of samples. Through developments in both technology and in bioinformatics to support metabolomics approaches, this situation is changing rapidly [20].

Metabolomics refers to comprehensive and non-selective analytical chemistry approaches aiming to provide a global description of all the metabolites present in a biofluid at a given time [7, 14, 23, 29, 55]. Metabolite contents of biofluids may be assessed through vibrational spectrometry platforms, including nuclear magnetic resonance (NMR), infrared spectroscopy (IR) or Fourier Transform IR (FTIR) or by capillary electrophoresis coupled either to ultraviolet absorbance detection (CE-UV) or to laser induced florescence detection (CE-LIF). In addition, there are a range of mass spectrometry (MS) based approaches, some without any chromatography e.g. flow injection electrospray ionisation MS (FIE-MS) and direct infusion MS (DIMS) and others coupled with a chromatographic step to first attempt to separate metabolites before detection such 
as gas chromatography (GC-MS), liquid chromatography (LC-MS) or high pressure liquid chromatography (HPLCMS). Any of these chromatographic steps may be followed by tandem MS or both NMR and MS [55]. The selection of the most suitable technology is generally a compromise between speed, selectivity and sensitivity.

Metabolomics datasets have specific characteristics which require appropriate statistical tools for their analysis. Indeed, where the intention is to measure simultaneously the entire metabolite content of biofluid samples collected from highly complex organisms (humans), the data produced by metabolomics experiments have enormous dimensionality (from 200 to 300 signals using GC-MS with time of flight detectors to about 2,000 using FIE-MS) and large biological variability $[13,30]$. Such dimensionality and variance demand the use of powerful, multivariate data analysis tools for sample classification or discrimination $[13,21,23,30]$. One of the best-known of these is principal component analysis (PCA), an unsupervised method which assesses natural clustering of sample classes and can be used to identify extreme outliers. For supervised analysis, typical multivariate algorithms used to separate treatment classes are linear discriminant analysis (LDA), partial least squares (PLS-DA), both discriminant analyses, and orthogonal projection to latent structures (OPLS), a form of regression analysis.

To date, metabolomics pipelines, which provide guidelines for all the steps from sample collection (with an appropriate study design), to the identification of two or more significantly different groups using pattern recognition statistics, has been applied to microbes, plants and some rodent models. Investigations with microbes have included the development of chemical taxonomy approaches to investigate the genetic diversity of fungal contaminants in food [52] or identity of bacterial species in mixed populations [57]. In plants, for example, metabolomics has been used to investigate possible unintended consequences in plants genetically engineered to exhibit novel enzyme activity [10]. In rodents, metabolomics approaches have been used in physiological evaluation, drug safety assessment, characterisation of genetically modified animal models of disease, and drug therapy monitoring [8, 34].

\section{Metabolomics approaches applied to measurement of dietary exposure}

In humans, metabolomics has been used mainly in studies focusing on diagnosis of disease $[9,11,15,25,32,39,40,56$, 58], mode of drug/toxin action [4, 31, 36, 42, 47], and characterisation of novel foods $[10,48]$. However, several recent commentary articles have suggested that metabolomics will have great value for nutritional studies $[12,16-20,38$, $62,65]$ and thus it is timely to exploit this technology platform to assess dietary exposure.

The first published study in which a metabolomics approach was described in a human nutrition experiment used NMR technology to monitor the effect of supplementing the diet with soy [53]. Only a small number of plasma samples were available and there was considerable inter-person variability but, despite these limitations, careful data pre-processing in combination with powerful discriminatory analysis grouped the samples into two main classes that reflected the dietary intervention.

Since then there have been a few studies using the power of metabolomics to link metabolite contents in human biofluids to acute or chronic dietary exposure. The study of urine samples obtained from healthy British and Swedish subjects revealed characteristic dietary and cultural features between the subjects of both countries, such as high trimethylamine- $N$-oxide (TMAO)-excretion in the Swedish population and high taurine-excretion, due to the Atkins diet [33]. Urine samples have also been used to investigate responses to ingestion of chamomile (Matricaria recutita) tea. Despite substantial inter-subject variation in metabolite profiles, clear differentiation between the samples obtained before and after chamomile ingestion was achieved with urinary excretion of hippurate and glycine being important discriminatory metabolites [60]. The effects of three experimental diets described as "vegetarian", "low meat", and "high meat" on urinary metabolite contents were also investigated [54]. PCA allowed differentiation of the characteristic metabolic signatures of the diets with creatine, carnitine, acetylcarnitine, and TMAO being elevated during the high-meat consumption period. Application of OPLS discriminant analysis allowed the low-meat diet and vegetarian diet signatures to be characterised, and $p$-hydroxyphenylacetate (a microbial-mammalian co-metabolite) was higher in the vegetarian than meat diet samples, signalling an alteration of the bacterial composition or metabolism in response to diet [54]. More recently, using urine samples from the large (4,630 participants) INTERMAP epidemiological study, involving 17 population samples in China, Japan, UK and USA, Holmes and collaborators have shown that a metabolomics approach can be used to distinguish East Asian from Western populations. In this study novel associations between urinary metabolites and blood pressure were discovered which suggested that both dietary factors per se and also altered gut microbial metabolism may be related to raised blood pressure [26]. Besides NMR-based technology, reports describing metabolite analysis using MS-based techniques in nutrition studies are emerging. For example, studies of polyphenol concentrations in human urine (using HPLC-tandem MS) after consumption of six different 
polyphenol-rich beverages showed that concentrations of chlorogenic acid, gallic acid, epicatechin, naringenin and hesperetin could be used as specific biomarkers to evaluate the consumption of coffee, wine, tea, cocoa and citrus juices, respectively [27].

\section{Selection of metabolomics approaches for characterising dietary exposure in humans}

The nutritional metabolomics studies undertaken in humans in the last 5-10 years have already demonstrated the ability of these techniques to measure known, or to discover new, compounds whose presence in blood or urine can be correlated with dietary exposure. This biomarker approach, which focuses on assessing specific biomarkers in biofluids to reveal the consumption of specific foods (e.g. TMAO, $\beta$-carotene or eicosapentanoic acid concentrations to identify high meat, vegetable or oily fish intakes, respectively), has limitations. Firstly, biomarker assays for a particular dietary component normally consists of the measurement of the concentration of one, or a few, blood/ urine metabolites using pure standards as a reference. Establishment of such assays presupposes sufficient background knowledge both of the areas of chemistry involved and the spectrum of metabolic responses to be expected from ingestion of the target food or food constituent to allow identification of the appropriate biomarker. For compounds such as anthocyanins for example, there is little reliable information on their absorption and metabolism in human subjects, and available studies have reported contradictory results [28]. Secondly, such approaches may demand specific extraction/purification procedures and use detailed, targeted, often low-throughput analytical procedures bespoke for each biomarker.

These targeted metabolomics analyses have been successfully applied in pharmacology, toxicology and medical screening [11, 15, 25, 32, 40, 42, 58], but are not entirely suitable for the characterisation of dietary exposure. The problem centres on the fact that currently well over $60 \%$ of the natural metabolites in raw food material have yet to be structurally characterised $[14,55]$ and, in consequence, the fate of such metabolites post-consumption are poorly understood. In addition, standards for many metabolites that potentially might be used as biomarkers are simply not available. With this lack of prior knowledge, the generation of hypotheses for the development of biomarkers indicative of exposure to specific food constituents is difficult [43, 45]. Further, any potential bioassays have to cope with the dynamic nature of metabolite concentrations in body fluids following food consumption. A logical approach to work within these limitations is to utilise metabolite "profiling" or "fingerprinting" techniques which allow the simultaneous monitoring of multiple components of blood/ urine whose, collective, relative behaviour may provide metabolic signals indicative of food intake. Such profiling/ fingerprinting assays should (1) be sensitive enough to survey signals from as many metabolites as possible in a non-biased way to allow the derivation of global metabolite profile/fingerprint patterns associated with specific features of diet, (2) not require any direct metabolite identification to develop a consistent metabolite fingerprint, (3) use metrics of relative signal ratios, rather than absolute concentration, to allow "normalisation" of fingerprints, (4) use data mining procedures that not only can discriminate between metabolite fingerprints but which can determine the most important signals responsible for the differences, (5) use analytical chemistry procedures in which the relevant fingerprint signals can be linked easily to specific metabolites.

Non-targeted metabolite profiling, using sensitive "time of flight" detectors, was used to analyse extracts of raw food materials $[10,64]$ and has utility for the detection of drug metabolites [45]. By attempting to profile all metabolite peaks detected automatically by instrument software, this approach is able to find metabolite differences between samples without any prior knowledge of which signals might be discriminatory. However, the use of a chromatographic step, to first attempt to separate metabolites before detection, requires exquisite control over the chromatographic process to obtain reproducibility and demands rigorous approaches to pre-process data to deconvolve, align and annotate peaks correctly [35].

A more "global" overview of total metabolite composition can be obtained from much more rapid and reproducible metabolite fingerprinting techniques which do not incorporate a chromatographic step. For example, FT-IR [22] and NMR [37, 61] generate global chemical fingerprints with little need for specialised sample preparation [23, 24]. However, these methods are less sensitive than FIE-MS [3] and, generally, require a further level of directed analysis to link any differences in wavenumber (FT-IR) or chemical shifts (NMR) to specific chemistry [23]. In contrast, fingerprinting techniques based on MS such as FIE-MS or DIMS offer the advantage that the measured "variables" [mass-to-charge $(\mathrm{m} / \mathrm{z})$ ratios] can be linked more directly to an individual metabolite using additional information on atomic mass [1, 2, 10, 42, 49, 52, 58];). FIE-MS fingerprints are developed following "soft" ionisation of the sample during injection over a period of 1-2 min and can be regarded as simplified images of total sample composition in that the $\mathrm{m} / \mathrm{z}$ ratios are compiled by integrating the levels of more than one metabolite (e.g. for isomers) that give a stable ion from each $m / z$. During soft ionisation the main products are charged versions of the parent molecule; fragmentation products are relatively rare 
and thus the identity of molecules producing signal at a specific $\mathrm{m} / \mathrm{z}$ can be investigated directly based on the predicted mass of the metabolite. The high throughput nature of FIE-MS is also apparent following data acquisition as fingerprint data require little, if any, pre-processing prior to analysis. The ability to analyse rapidly a large number of samples with minimal data processing provides desirable attributes for first pass sample analysis in large scale epidemiological studies.

\section{Perspectives}

Such high-throughput, non-targeted metabolite fingerprinting using FIE-MS has been successfully applied and validated in the context of the dynamic interaction between a pathogen and its host, where a time series of leaf tissues were collected from Brachypodium leaves after infection with the fungal pathogen Magnaporthe grisea [3, 13, 41]. We are now applying this analytical approach to characterise dietary exposure in humans through measurements on blood and urine. In the MEDE Study (MEtabolomics to characterise Dietary Exposure), blood and urine samples have been collected from healthy volunteers in the fasted state and after consumption of carefully designed test meals. The results from the first phase of the MEDE Study are promising showing that generated metabolite fingerprints (1) were reproducible within individuals, (2) discriminated clearly between fasting and fed samples, and (3) displayed an overall variance dominated by sample treatment class and not by gender or individual (Favé et al. Unpublished data). The next phase of the MEDE Study will investigate the metabolite fingerprints in biofluids from volunteers exposed to specific test foods including an oily fish, a wholegrain cereal product, a green vegetable and a fruit. The MEDE Study is designed to provide proof of principle that metabolomics approaches can be used to generate novel biomarkers of dietary intake in circumstances where the biofluids have been collected at 3-4 times over a few hours after consumption of the test meal. Metabolomics might also be useful in characterising habitual dietary exposure but here the challenge is likely to be considerably greater. This will require additional systematic research including investigation of the kinetics of metabolite transfers between available biofluid pools (e.g. blood, urine and saliva) and sites of storage or sequestration e.g. adipose tissue or bone of the metabolites to identify metabolites with long whole body half-lives which will be candidates for assessment of sustained (habitual) exposure.

Acknowledgment The MEDE Study is supported by the UK Food Standard Agency (Project N05073).

\section{References}

1. Aharoni A, Ric de Vos CH, Verhoeven HA, Maliepaard CA, Kruppa G, Bino R, Goodenowe DB (2002) Nontargeted metabolome analysis by use of Fourier Transform Ion Cyclotron Mass Spectrometry. Omics 6(3):217-234

2. Allen J, Davey HM, Broadhurst D, Heald JK, Rowland JJ, Oliver SG, Kell DB (2003) High-throughput classification of yeast mutants for functional genomics using metabolic footprinting. Nat Biotechnol 21(6):692-696

3. Beckmann M, Parker D, Enot DP, Duval E, Draper J (2008) High-throughput, nontargeted metabolite fingerprinting using nominal mass flow injection electrospray mass spectrometry. Nat Protoc 3(3):486-504

4. Beckonert O, Bollard ME, Ebbels TMD, Keun HC, Antti H, Holmes E, Lindon JC, Nicholson JK (2003) NMR-based metabonomic toxicity classification: hierarchical cluster analysis and k-nearest-neighbour approaches. Anal Chim Acta 490(1-2):3-15

5. Bingham SA (2002) Biomarkers in nutritional epidemiology. Public Health Nutr 5(6A):821-827

6. Bingham SA, Gill C, Welch A, Cassidy A, Runswick SA, Oakes S, Lubin R, Thurnham DI, Key TJ, Roe L, Khaw KT, Day NE (1997) Validation of dietary assessment methods in the UK arm of EPIC using weighed records, and 24-h urinary nitrogen and potassium and serum vitamin $\mathrm{C}$ and carotenoids as biomarkers. Int J Epidemiol 26(Suppl 1):S137-S151

7. Bino RJ, Hall RD, Fiehn O, Kopka J, Saito K, Draper J, Nikolau BJ, Mendes P, Roessner-Tunali U, Beale MH, Trethewey RN, Lange BM, Wurtele ES, Sumner LW (2004) Potential of metabolomics as a functional genomics tool. Trends Plant Sci 9(9):418-425

8. Bollard ME, Stanley EG, Lindon JC, Nicholson JK, Holmes E (2005) NMR-based metabonomic approaches for evaluating physiological influences on biofluid composition. NMR Biomed 18(3):143-162

9. Brindle JT, Antti H, Holmes E, Tranter G, Nicholson JK, Bethell HW, Clarke S, Schofield PM, McKilligin E, Mosedale DE, Grainger DJ (2002) Rapid and noninvasive diagnosis of the presence and severity of coronary heart disease using 1H-NMRbased metabonomics. Nat Med 8(12):1439-1444

10. Catchpole GS, Beckmann M, Enot DP, Mondhe M, Zywicki B, Taylor J, Hardy N, Smith A, King RD, Kell DB, Fiehn O, Draper J (2005) Hierarchical metabolomics demonstrates substantial compositional similarity between genetically modified and conventional potato crops. Proc Natl Acad Sci USA 102(40):1445814462

11. Chace DH, Kalas TA, Naylor EW (2003) Use of tandem mass spectrometry for multianalyte screening of dried blood specimens from newborns. Clin Chem 49(11):1797-1817

12. Davis CD, Milner J (2004) Frontiers in nutrigenomics, proteomics, metabolomics and cancer prevention. Mutat Res 551(1-2): 51-64

13. Enot DP, Lin W, Beckmann M, Parker D, Overy DP, Draper J (2008) Preprocessing, classification modeling and feature selection using flow injection electrospray mass spectrometry metabolite fingerprint data. Nat Protoc 3(3):446-470

14. Fiehn O (2002) Metabolomics-the link between genotypes and phenotypes. Plant Mol Biol 48(1-2):155-171

15. Fu X, Iga M, Kimura M, Yamaguchi S (2000) Simplified screening for organic acidemia using GC/MS and dried urine filter paper: a study on neonatal mass screening. Early Hum Dev 58(1):41-55

16. German AJ, Watkins SM (2004) Metabolic assessment-a key to nutritional strategies for health. Trends Food Sci Technol 15(11):541-549 
17. German JB, Roberts MA, Fay L, Watkins SM (2002) Metabolomics and individual metabolic assessment: the next great challenge for nutrition. J Nutr 132(9):2486-2487

18. German JB, Roberts MA, Watkins SM (2003) Genomics and metabolomics as markers for the interaction of diet and health: lessons from lipids. J Nutr 133(6 Suppl 1):2078S-2083S

19. German JB, Roberts MA, Watkins SM (2003) Personal metabolomics as a next generation nutritional assessment. J Nutr 133(12):4260-4266

20. Gibney MJ, Walsh M, Brennan L, Roche HM, German B, van Ommen B (2005) Metabolomics in human nutrition: opportunities and challenges. Am J Clin Nutr 82(3):497-503

21. Goodacre R, Broadhurst D, Smilde AK, Kristal BS, Baker JD, Beger R, Bessant C, Connor S, Capuani G, Craig A, Ebbels T, Kell DB, Manetti C, Newton J, Paternostro G, Somorjai R, Sjöström M, Trygg J, Wulfert F (2007) Proposed minimum reporting standards for data analysis in metabolomics. Metabolomics 3(3):231-241

22. Goodacre R, Shann B, Gilbert RJ, Timmins EM, McGovern AC, Alsberg BK, Kell DB, Logan NA (2000) Detection of the dipicolinic acid biomarker in Bacillus spores using Curie-point pyrolysis mass spectrometry and Fourier transform infrared spectroscopy. Anal Chem 72(1):119-127

23. Goodacre R, Vaidyanathan S, Dunn WB, Harrigan GG, Kell DB (2004) Metabolomics by numbers: acquiring and understanding global metabolite data. Trends Biotechnol 22(5):245-252

24. Griffin JL (2003) Metabonomics: NMR spectroscopy and pattern recognition analysis of body fluids and tissues for characterisation of xenobiotic toxicity and disease diagnosis. Curr Opin Chem Biol 7(5):648-654

25. Halket JM, Przyborowska A, Stein SE, Mallard WG, Down S, Chalmers RA (1999) Deconvolution gas chromatography/mass spectrometry of urinary organic acids-potential for pattern recognition and automated identification of metabolic disorders. Rapid Commun Mass Spectrom 13(4):279-284

26. Holmes E, Loo RL, Stamler J, Bictash M, Yap IK, Chan Q, Ebbels T, De Iorio M, Brown IJ, Veselkov KA, Daviglus ML, Kesteloot H, Ueshima H, Zhao L, Nicholson JK, Elliott P (2008) Human metabolic phenotype diversity and its association with diet and blood pressure. Nature 453(7193):396-400

27. Ito H, Gonthier MP, Manach C, Morand C, Mennen L, Remesy C, Scalbert A (2005) Polyphenol levels in human urine after intake of six different polyphenol-rich beverages. Br J Nutr 94(4):500-509

28. Kay CD, Mazza G, Holub BJ, Wang J (2004) Anthocyanin metabolites in human urine and serum. Br J Nutr 91(6):933-942

29. Kell DB (2004) Metabolomics and systems biology: making sense of the soup. Curr Opin Microbiol 7(3):296-307

30. Kemsley EK, Le Gall G, Dainty JR, Watson AD, Harvey LJ, Tapp HS, Colquhoun IJ (2007) Multivariate techniques and their application in nutrition: a metabolomics case study. Br J Nutr $98(1): 1-14$

31. Keun HC (2006) Metabonomic modeling of drug toxicity. Pharmacol Ther 109(1-2):92-106

32. Kim KR, Park HG, Paik MJ, Ryu HS, Oh KS, Myung SW, Liebich HM (1998) Gas chromatographic profiling and pattern recognition analysis of urinary organic acids from uterine myoma patients and cervical cancer patients. J Chromatogr B Biomed Sci Appl 712(1-2):11-22

33. Lenz EM, Bright J, Wilson ID, Hughes A, Morrisson J, Lindberg $\mathrm{H}$, Lockton A (2004) Metabonomics, dietary influences and cultural differences: a $1 \mathrm{H}$ NMR-based study of urine samples obtained from healthy British and Swedish subjects. J Pharm Biomed Anal 36(4):841-849

34. Lindon JC, Holmes E, Bollard ME, Stanley EG, Nicholson JK (2004) Metabonomics technologies and their applications in physiological monitoring, drug safety assessment and disease diagnosis. Biomarkers 9(1):1-31

35. Lisec J, Schauer N, Kopka J, Willmitzer L, Fernie AR (2006) Gas chromatography mass spectrometry-based metabolite profiling in plants. Nat Protoc 1(1):387-396

36. Nicholson JK, Connelly J, Lindon JC, Holmes E (2002) Metabonomics: a platform for studying drug toxicity and gene function. Nat Rev Drug Discov 1(2):153-161

37. Nicholson JK, Wilson ID (2003) Opinion: understanding 'global' systems biology: metabonomics and the continuum of metabolism. Nat Rev Drug Discov 2(8):668-676

38. Noguchi Y, Sakai R, Kimura T (2003) Metabolomics and its potential for assessment of adequacy and safety of amino acid intake. J Nutr 133(6 Suppl 1):2097S-2100S

39. Odunsi K, Wollman RM, Ambrosone CB, Hutson A, McCann SE, Tammela J, Geisler JP, Miller G, Sellers T, Cliby W, Qian F, Keitz B, Intengan M, Lele S, Alderfer JL (2005) Detection of epithelial ovarian cancer using $1 \mathrm{H}-\mathrm{NMR}-$ based metabonomics. Int J Cancer 113(5):782-788

40. Ohdoi C, Nyhan WL, Kuhara T (2003) Chemical diagnosis of Lesch-Nyhan syndrome using gas chromatography-mass spectrometry detection. J Chromatogr B Analyt Technol Biomed Life Sci 792(1):123-130

41. Parker D, Beckmann M, Enot DP, Overy DP, Rios ZC, Gilbert M, Talbot N, Draper J (2008) Rice blast infection of Brachypodium distachyon as a model system to study dynamic host/pathogen interactions. Nat Protoc 3(3):435-445

42. Pelander A, Ojanpera I, Laks S, Rasanen I, Vuori E (2003) Toxicological screening with formula-based metabolite identification by liquid chromatography/time-of-flight mass spectrometry. Anal Chem 75(21):5710-5718

43. Pham-Tuan H, Kaskavelis L, Daykin CA, Janssen HG (2003) Method development in high-performance liquid chromatography for high-throughput profiling and metabonomic studies of biofluid samples. J Chromatogr B Analyt Technol Biomed Life Sci 789(2):283-301

44. Plakke T, Berkel J, Beynen AC, Hermus RJ, Katan MB (1983) Relationship between the fatty acid composition of the diet and that of the subcutaneous adipose tissue in individual human subjects. Hum Nutr Appl Nutr 37(5):365-372

45. Plumb RS, Stumpf CL, Granger JH, Castro-Perez J, Haselden JN, Dear GJ (2003) Use of liquid chromatography/time-of-flight mass spectrometry and multivariate statistical analysis shows promise for the detection of drug metabolites in biological fluids. Rapid Commun Mass Spectrom 17(23):2632-2638

46. Qian GS, Ross RK, Yu MC, Yuan JM, Gao YT, Henderson BE, Wogan GN, Groopman JD (1994) A follow-up study of urinary markers of aflatoxin exposure and liver cancer risk in Shanghai, People's Republic of China. Cancer Epidemiol Biomarkers Prev 3(1):3-10

47. Robertson DG (2005) Metabonomics in toxicology: a review. Toxicol Sci 85(2):809-822

48. Roessner U, Wagner C, Kopka J, Trethewey RN, Willmitzer L (2000) Technical advance: simultaneous analysis of metabolites in potato tuber by gas chromatography-mass spectrometry. Plant J 23(1):131-142

49. Scholz M, Gatzek S, Sterling A, Fiehn O, Selbig J (2004) Metabolite fingerprinting: detecting biological features by independent component analysis. Bioinformatics 20(15):2447-2454

50. Seale JL, Klein G, Friedmann J, Jensen GL, Mitchell DC, Smiciklas-Wright H (2002) Energy expenditure measured by doubly labeled water, activity recall, and diet records in the rural elderly. Nutrition 18(7-8):568-573

51. Smedman AE, Gustafsson IB, Berglund LG, Vessby BO (1999) Pentadecanoic acid in serum as a marker for intake of milk fat: 
relations between intake of milk fat and metabolic risk factors. Am J Clin Nutr 69(1):22-29

52. Smedsgaard J, Frisvad JC (1996) Using direct electrospray mass spectrometry in taxonomy and secondary metabolite profiling of crude fungal extracts. J Microbiol Methods 25(1):5-17

53. Solanky KS, Bailey NJ, Beckwith-Hall BM, Davis A, Bingham S, Holmes E, Nicholson JK, Cassidy A (2003) Application of biofluid $1 \mathrm{H}$ nuclear magnetic resonance-based metabonomic techniques for the analysis of the biochemical effects of dietary isoflavones on human plasma profile. Anal Biochem 323(2):197204

54. Stella C, Beckwith-Hall B, Cloarec O, Holmes E, Lindon JC, Powell J, van der Ouderaa F, Bingham S, Cross AJ, Nicholson JK (2006) Susceptibility of human metabolic phenotypes to dietary modulation. J Proteome Res 5(10):2780-2788

55. Sumner LW, Mendes P, Dixon RA (2003) Plant metabolomics: large-scale phytochemistry in the functional genomics era. Phytochemistry 62(6):817-836

56. t Hart BA, Vogels JT, Spijksma G, Brok HP, Polman C, van der Greef J (2003) 1H-NMR spectroscopy combined with pattern recognition analysis reveals characteristic chemical patterns in urines of MS patients and non-human primates with MS-like disease. J Neurol Sci 212(1-2):21-30

57. Vaidyanathan S, Kell DB, Goodacre R (2002) Flow-injection electrospray ionization mass spectrometry of crude cell extracts for high-throughput bacterial identification. J Am Soc Mass Spectrom 13(2):118-128

58. Valianpour F, Selhorst JJ, van Lint LE, van Gennip AH, Wanders RJ, Kemp S (2003) Analysis of very long-chain fatty acids using electrospray ionization mass spectrometry. Mol Genet Metab 79(3):189-196

59. Wang C, Baumgartner RN, Yang D, Slattery ML, Murtaugh MA, Byers T, Hines LM, Giuliano AR, Baumgartner KB (2009) No evidence of association between breast cancer risk and dietary carotenoids, retinols, vitamin $\mathrm{C}$ and tocopherols in Southwestern Hispanic and non-Hispanic white women. Breast Cancer Res Treat 114(1):137-145

60. Wang Y, Tang H, Nicholson JK, Hylands PJ, Sampson J, Holmes E (2005) A metabonomic strategy for the detection of the metabolic effects of chamomile (Matricaria recutita L.) ingestion. J Agric Food Chem 53(2):191-196

61. Ward JL, Harris C, Lewis J, Beale MH (2003) Assessment of $1 \mathrm{H}$ NMR spectroscopy and multivariate analysis as a technique for metabolite fingerprinting of Arabidopsis thaliana. Phytochemistry 62(6):949-957

62. Watkins SM, German JB (2002) Toward the implementation of metabolomic assessments of human health and nutrition. Curr Opin Biotechnol 13(5):512-516

63. Weber JL, Reid PM, Greaves KA, DeLany JP, Stanford VA, Going SB, Howell WH, Houtkooper LB (2001) Validity of selfreported energy intake in lean and obese young women, using two nutrient databases, compared with total energy expenditure assessed by doubly labeled water. Eur J Clin Nutr 55(11):940 950

64. Weckwerth W, Wenzel K, Fiehn O (2004) Process for the integrated extraction, identification and quantification of metabolites, proteins and RNA to reveal their co-regulation in biochemical networks. Proteomics 4(1):78-83

65. Whitfield PD, German AJ, Noble PJ (2004) Metabolomics: an emerging post-genomic tool for nutrition. Br J Nutr 92(4):549555

66. Wolk A, Vessby B, Ljung H, Barrefors P (1998) Evaluation of a biological marker of dairy fat intake. Am J Clin Nutr 68(2):291295 